\title{
Les reliures des livres d'Heures manuscrits de l'Université McGill et la reliure gothique d’origine du McGill, MS 101
}

\author{
GENEVIÈVE SAMSON \\ Bibliothèque et Archives Canada
}

Une reliure sert avant tout à protéger l'ouvrage qu'elle recouvre. Elle doit aussi être considérée comme un élément autonome qui a son esthétique, ses techniques et son histoire propres. Cet article présentera d'abord, de manière générale, les reliures des neuf livres d'Heures manuscrits conservés à McGill, dont certaines sont d'origine et d'autres furent restaurées entre le XVII et la fin du XX ${ }^{e}$ siècle. En second lieu, la remarquable reliure originale du manuscrit McGill, MS 101 sera décrite en détail. Cette reliure est la plus représentative du corpus au pour son vocabulaire stylistique de la reliure de la deuxième moitié $d u X V^{e}$ siècle avec ses ais, ses fermoirs en laiton torsadé et son cuir décoré à froid de fers dit monastiques. L'analyse codicologique de ces livres d'Heures contribue à l'archéologie du livre médiéval et s'inscrit dans une perspective de mise en valeur de la reliure ancienne en Occident entreprise par de grandes bibliothèques européennes.

A book's binding, above all else, serves to protect the work inside. It must also be considered as an autonomous element that has its own aesthetic, techniques, and history. This article will present, in a general way, the bindings of nine Books of Hours preserved at McGill, some of which are original and others that were restored between the seventeenth century and the end of the twentieth. Secondly, the remarkable original binding of manuscript McGill, MS 101 will be described in detail. This binding is the most representative of the corpus when considering the stylistic vocabulary of book bindings during the second half of the fifteenth century with its panels, braided brass fasteners, and leather which was decorated freehand with a monastic design. The codicological analysis of these Books of Hours contributes to the archeology of the medieval book and follows a perspective of the development of ancient book binding in the West undertaken by great European libraries.

$S_{\text {aussi être considérée comme un élément autonome qui a son histoire, }}^{\text {i une reliure sert avant tout à protéger l'ouvrage quelle recouvre, elle peut }}$ sa technique et ses fonctions. L'étude que nous présentons ici porte sur des reliures conservées dans les collections de McGill. En effet, les Livres rares et collections spécialisées de McGill possèdent douze livres d'Heures, dont neuf sont manuscrits et trois imprimés. L'objet de cet article est de décrire les reliures ou leurs vestiges de manière sommaire pour huit de ces manuscrits puis de proposer une description poussée de la reliure du McGill, MS 101 qui est à la 
fois le seul manuscrit à conserver sa reliure originale et à ne comporter aucune enluminure.

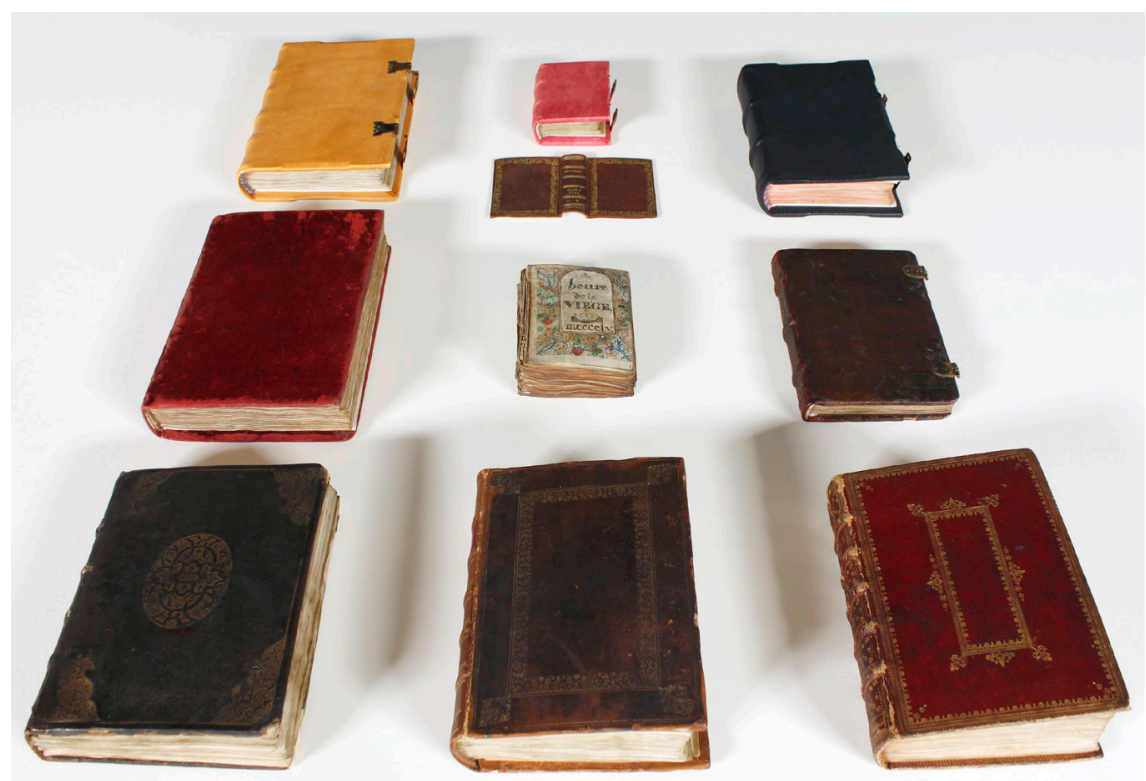

Fig. 1. Le corpus des neuf reliures. Première rangée, de gauche à droite : McGill, MS 97, McGill, MS 98 et McGill, MS 155 ; deuxième rangée, de gauche à droite : McGill, MS 154, McGill, MS 158 et McGill, MS 101 ; troisième rangée, de gauche à droite : McGill, MS 100, McGill, MS 109 et McGill, MS $156^{1}$.

1. L’auteur exprime sa gratitude à Sarah Severson et Greg Houtson du service de Digitization and Delivery Department de McGill University Library pour leur aide et assistance pour cette photographie et à la Collection des livres rares et des collections spécialisées de l'Université McGill pour la reproduire. Les tableaux et les autres photographies sont de l'auteur. Ces dernières ont été prises avec la permission de la Collection des livres rares et des collections spécialisées de l'Université McGill que nous remercions. Cet article s'inscrit dans le projet de recherche sur les livres d'Heures conservés au Québec financé par le CRSH (2014-2018) et sous la direction de Brenda Dunn-Lardeau. 


\section{Description générale des reliures du corpus}

Aux fins de la description générale du corpus de neuf manuscrits sous l'angle de la reliure, nous avons d'abord regroupé huit des neuf reliures (ou leurs vestiges) conservées aux Livres rares et collections spécialisées de McGill selon certaines caractéristiques : le premier groupe est constitué de trois livres d'Heures pour lesquels de nouvelles reliures ont été réalisées entre le $\mathrm{XVI}^{e}$ et le $\mathrm{XVII}{ }^{e}$ siècle (McGill, MS 97, McGill, MS 98 et McGill, MS 155); le second groupe comporte trois reliures réalisées au $\mathrm{XX}^{\mathrm{e}}$ siècle et qui pastichent le style gothique (McGill, MS 100, McGill, MS 109 et McGill, MS 156). Dans le troisième groupe se retrouve une reliure en velours rouge (McGill, MS 154), dont la date de réfection est difficile à préciser et un manuscrit (McGill, MS 158) qui a été démonté sans sa reliure.

Nous présenterons à la fin de cette description générale un tableau codicologique sommaire de l'ensemble du corpus incluant le neuvième manuscrit, le McGill, MS 101, cas particulier sur lequel nous reviendrons toutefois en détail dans la deuxième partie pour rendre justice à sa reliure originale.

\subsection{Les livres d'Heures McGill, MS 97, McGill, MS 98 et McGill, MS 155 : réfections entre les $\mathrm{XVI}^{\mathrm{e}}$ et $\mathrm{XVIII}{ }^{\mathrm{e}}$ siècles}

Les trois manuscrits du premier groupe sont à rapprocher par l'époque de leur reliure : celle du manuscrit McGill, MS 97 a été réalisée fin XVIe-début XVIIe siècle et les reliures pour les manuscrits McGill, MS 155 et MS 98 ont été réalisées entre la fin du XVII et la fin du XVIII siècle $^{2}$.

1.1.1. Manuscrit McGill, MS 97, livre d'Heures à l'usage de Sarum (Salisbury), Bruges, deuxième moitié du $\mathrm{XV}^{\mathrm{e}}$ siècle

Cette reliure, réalisée au tournant des XVI ${ }^{\mathrm{e}} \mathrm{XVII}{ }^{\mathrm{e}}$ siècles, comme nous l'avions mentionné, est de veau brun foncé sur carton, ornée avec une plaque centrale, frappée d'or, d'un grand motif central de forme ovale, azuré et complété par des écoinçons. Le dos est à quatre nerfs apparents avec décor aux entre-nerfs 
alors que le codex, originalement cousu sur trois lanières de cuir ou septains de chanvre, a été complètement démonté puis recousu sur deux rubans de lin, une technique utilisée vers la fin du XIX ${ }^{\mathrm{e}}$ siècle. Les coiffes ont été retouchées et consolidées avec un cuir de veau, mais sans l'ajout de tranchefiles. Deux éléments structuraux du livre d'Heures ne concordent pas avec le format du codex, soit la couture et les plats. Pour ce qui est de la couture, deux styles d'entailles sont présentes dans les fonds des cahiers : une première, des doubles encoches faites par un couteau de relieur (XVe siècle) et une seconde, des trous ronds faits soit par une scie à grecquer, soit une aiguille pour coudre autour des rubans de lin (fin XIX ${ }^{\mathrm{e}}$ siècle). Nous notons aussi la présence de quatre septains à nerfs fendus toujours encastrés dans le cuir du dos du livre. Ces derniers ne correspondent pas aux emplacements des deux styles d'entailles présents dans le fond des cahiers. Nous nous retrouvons avec trois témoignages structuraux : un codex datant du $\mathrm{XV}^{\mathrm{e}}$ siècle avec des entailles pour trois nerfs fendus, une reliure datant de la fin du XVIe ou du milieu du XVII e siècle contenant toujours la présence de quatre nerfs fendus et enfin, une couture datant de la fin du XIX ou du début $\mathrm{XX}^{\mathrm{e}}$ siècle. Si nous considérons ensuite le format du codex, nous constatons qu'il n'y a aucune chasse et que le parchemin déborde des plats des trois côtés. C'est ce dernier élément qui nous éclaire : les reliures datant de cette période ayant normalement des chasses, nous nous trouvons donc ici devant un cas de reliure réutilisée, c'est-à-dire un cas de réemploi de la reliure d'un autre ouvrage dont la source nous est inconnue. Peut-être surprend-on ici une astuce du relieur pour s'éviter du travail, ou tromper un éventuel acquéreur, en réemployant une reliure retirée d'un ouvrage, sans doute de moindre importance, afin d'en tirer un meilleur bénéfice pécuniaire.

1.1.2. Manuscrit McGill, MS 98, livre d'Heures à l'usage de Sarum, Bruges, fin du XVe siècle, vers 1460-1470

Ce deuxième livre d'Heures possède une reliure en veau brun sur carton, ornée d'un décor à la roulette et aux fers du XVII e siècle. Le livre a connu une restauration à la fin du $\mathrm{XIX}^{\mathrm{e}}$ ou au début du $\mathrm{XX}^{\mathrm{e}}$ siècle. Il a gardé ses plats anciens, mais le dos a été remplacé par un nouveau dos en cuir brun avec des coiffes contenant un septain pour imiter des tranchefiles. Il ne contient aucun décor, mais le titre est en cuir rouge. Il faut mentionner la présence de contregarde et de garde volante simple, faite d'un papier moderne sans filigrane. Le 
corps d'ouvrage a été démonté puis recousu sur cinq septains de chanvre simple, les premiers et derniers cahiers remontés en style de surjet - technique visant à consolider l'ensemble, mais rendant souvent difficile l'ouverture d'un volume.

1.1.3. Manuscrit McGill, MS 155, livre d'Heures à l'usage de Rome, France, fin du XVe siècle

Cette reliure, datant de la fin du XVII ${ }^{e}$ ou du début du XVIII e siècle, est dite " à la Du Seuil $»^{3}$ puisqu'elle présente les caractéristiques suivantes : l'œuvre est reliée en maroquin rouge de grande qualité ; son décor comprend un encadrement de filets sur les plats, très proches des bords, et un encadrement intérieur similaire sur les plats, avec un fleuron aux quatre coins, un second fleuron le long de l'encadrement central et un décor de fleurons sur un dos à cinq nerfs apparents ; des tranchefiles sont montées sur un bâti fait d'un mince carton et décorées avec des fils de soie de couleurs jaune et rouge. Deux autres éléments intéressants sont à signaler : la contre-garde et la garde volante sont composées d'un papier à la colle d'un motif complexe et d'une grande qualité, avec ses couleurs toujours flamboyantes, tandis qu'un papier à la cuve est collé à l'endos du papier décoré. Ils sont pliés à cheval et cousus à la couture. À l'endos de la garde volante, court un filigrane avec la marque du raisin à la tige très longue comprenant vingt-cinq grains de grappe. C'est un modèle très répandu depuis le $\mathrm{XV}^{\mathrm{e}}$ siècle, mais sans la contremarque contenant les initiales ou le nom du papetier, il est toutefois difficile d'identifier le moulin d'où provient ce papier ainsi que la date de fabrication de ce papier à la cuve.

\subsection{Les reliures «à l'identique " du XVIe siècle : les livres d'Heures McGill, MS 100, McGill, MS 109 et McGill, MS 156}

Selon les dossiers de collection de l'Université McGill, nous savons que ces trois livres d'Heures ont été restaurés en 1996 par un même restaurateur, Madame Terry Rutheford. Les photographies des dossiers respectifs nous montrent que les dernières réfections datent de la fin du XVIII ${ }^{e}$ ou du début du XIX ${ }^{\mathrm{e}}$ siècle.

3. Roger Devauchelle, La reliure: Recherches historiques, techniques et biographiques sur la reliure française (France : Édition Filigranes, 1995), 124. 
Les matériaux de couvrure pour les manuscrits MS 109 et MS 100 sont le parchemin, teinté rose vif pour le manuscrit MS 109 et ocre pour le manuscrit MS 100. Pour le manuscrit MS 156, la couvrure est d'un cuir de chèvre de couleur bleu foncé. Quant aux plats des manuscrits MS 100, MS 109 et MS 156, ceux-ci sont en bois, biseautés sur les trois côtés, sauf pour le manuscrit MS 109. Les fermoirs ne sont pas d'origine, mais complètent le style gothique voulu par le restaurateur : un œillet et une mortaise pour le manuscrit MS 109 ; une agrafe et une contre-agrafe pour les manuscrits MS 100 et MS 156. Le départ des fermoirs des manuscrits MS 109 et MS 156 se fait du plat inférieur, tandis que celui du manuscrit MS 100 part du plat supérieur. Les nouvelles coutures, respectueuses du style gothique, sont faites de lanières de peau fendue, deux pour les manuscrits MS 109 et MS 156 et quatre pour le manuscrit MS 100. Les tranchefiles des manuscrits MS 100, MS 109 et MS 156 sont exécutées sur un septain de chanvre et couvert d'un fil de lin. Les bâtis des manuscrits MS 100 et MS 156 sont agrémentés d'une décoration secondaire, dite en chevron, de couleur rose et bleue, une fantaisie du restaurateur. Malgré ce façonnage habile moderne sur un livre ancien, le résultat final de ces nouvelles reliures est une réplique de l'original. Ce sont maintenant des objets composites, entre pastiche et fac-similé.

\subsection{Le livre d'Heures McGill, MS 154 relié en velours et le livre d'Heures démonté, le McGill, MS 158}

\subsubsection{Manuscrit McGill, MS 154, livre d'Heures à l'usage de Rome, Bourgogne,} vers $1480-1490$

La septième reliure est la seule en tissu. Il s'agit d'un velours rouge monté sur des ais de bois, non biseautés, le dos du volume est lisse et les tranchefiles simples sont de soie. Plusieurs éléments structuraux nous donnent des indices quant aux dates de réfection de ce livre. Premièrement, la couture : des entailles rondes pour les supports. Cela signifie que ces entailles ont été faites non par un couteau, mais par une scie à grecquer. Cette technique, utilisée à partir du $\mathrm{XVI}^{\mathrm{e}}$ siècle, mais qui se généralise au XIXe siècle, permet de loger les ficelles et les chaînettes à l'intérieur des cahiers et donne ainsi un dos plus lisse $\mathrm{e}^{4}$. 
Deuxièmement, les contre-gardes de papier à la cuve sont collées aux plats et non pas cousues de manière continue avec le texte. Cette opération s'exécute à la fin du montage du corps d'ouvrage, non plus au moment de la couture. Un autre élément de datation est la présence d'un filigrane sur les gardes flottantes. Il s'agit d'une grande fleur de lis sur écus polonais, accompagné du chiffre quatre et des initiales WR pour Wendelin Riehel. Ce modèle très répandu fut utilisé dès la fin du XVI e et ce, jusqu'à la fin du XVIII siècle ${ }^{5}$. Et que dire de ces ais de bois qui nous ramènent aux XVI ${ }^{e}$ et XVII ${ }^{e}$ siècles ? La possibilité, encore une fois, d'une réutilisation de plats, en contradiction avec cette couvrure en velours, constitue une énigme que nous pourrons peut-être résoudre au fil de la recherche.

1.3.2. Manuscrit McGill, MS 158, livre d'Heures de la fin du XVe siècle, nord de la France ou Flandre (?)

Ce huitième livre d'Heures a subi un démontage agressif et la plupart des feuillets ne sont plus rattachés. Malgré tout, un vestige d'un élément structurel reste apparent : la couture. On trouve des traces de deux différents styles d'entailles, une au couteau pour une couture sur lanière ou septain, l'autre pour une couture sur rubans.

5. Raymond Gaudriault, Filigranes et autres caractéristiques des papiers fabriqués en France aux XVII et XVIII siècles (Paris : CNRS, 1995), 132. 


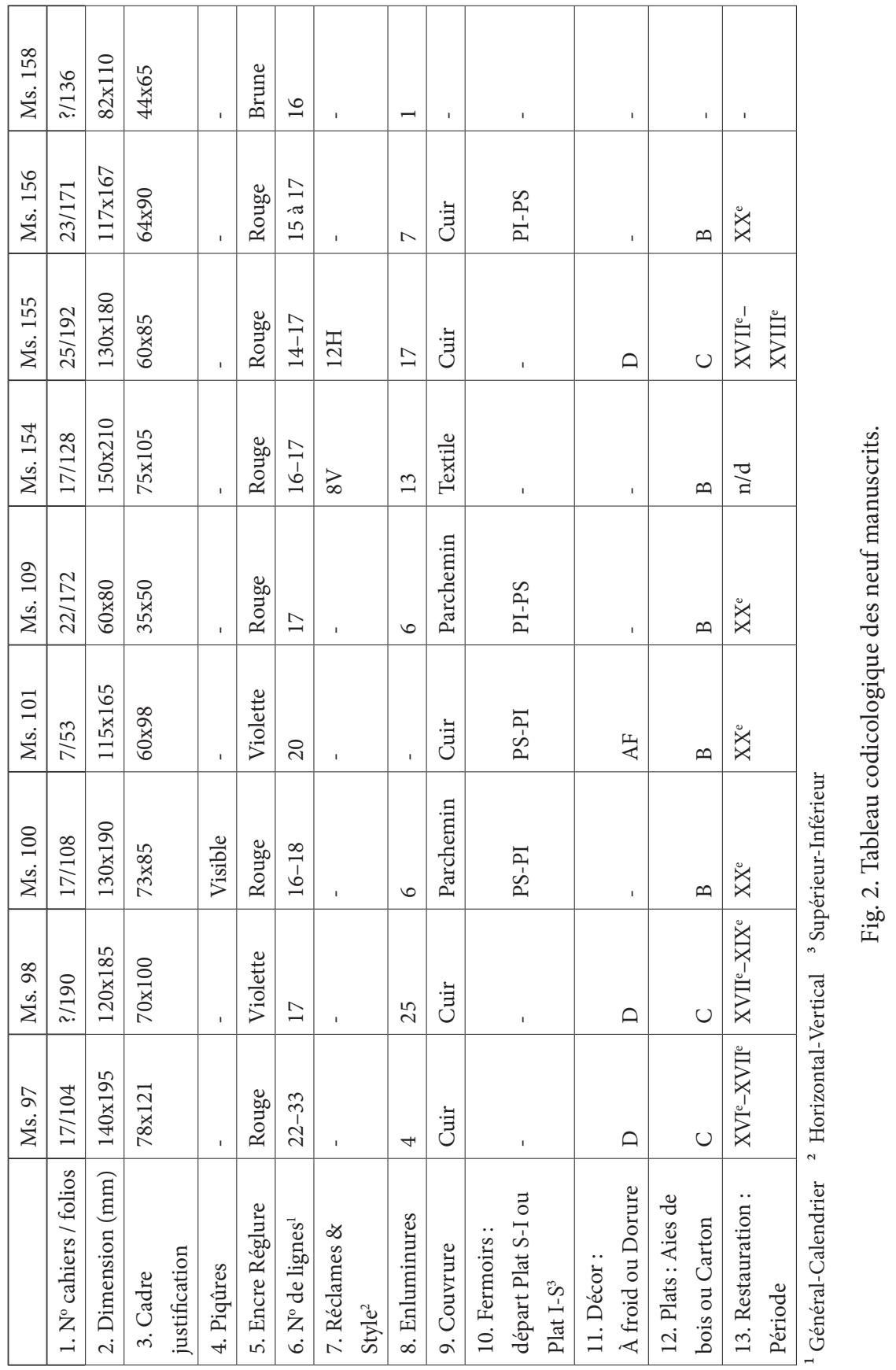


Dans ce tableau, on trouve en abscisse, la liste numérique des neufs manuscrits, et, en ordonnée, les éléments suivants : le nombre de cahiers et de folios, la dimension des plats suivie par le cadre de justification en millimètres, ensuite les piqûres, l'encre utilisée pour la réglure, le nombre de lignes, les réclames et leur position et le nombre d'enluminures par livre. Ensuite viennent les éléments de reliure, la couvrure (cuir, parchemin ou textile), puis les fermoirs avec les départs, des plats supérieur ou inférieur. Suivent le décor ainsi que le matériel utilisé pour les plats (le bois ou le carton) et finalement, la date de la dernière reliure.

\section{La reliure gothique du $\mathrm{XV}^{\mathrm{e}}$ siècle du livre d'Heures McGill, MS 101}

Nous procéderons maintenant à notre étude de cas soit, d'abord, l'examen spécifique de la reliure du manuscrit McGill, MS 101, la seule reliure d'origine de l'ensemble du corpus, puis, à la collation des cahiers du manuscrit et à son analyse codicologique.

\subsection{Description matérielle du manuscrit McGill, MS 101, livre d'Heures à l'usage de Rome, Flandre, vers 1450-1480}

Souvent dans les dossiers de collection, nous trouvons des notes concernant la description matérielle du document. Dans le cas du manuscrit qui nous occupe, nous avons retrouvé dans le dossier de recherche du manuscrit McGill, MS 101 une fiche descriptive dactylographiée de D. Hatzopoulos qui reprend la description qu'a donnée de Ricci dans son Census ${ }^{6}$. Cette fiche contientle numéro attribué au manuscrit ainsi que la source et la date de l'acquisition. Également, collée sur la page de garde du plat inférieur, se retrouve une notice découpée $\mathrm{du}$ catalogue du vendeur dans laquelle on trouve une première description sommaire de la reliure. Ces trois descriptions concordent. Le manuscrit McGill, MS 101 est de dimension et de présentation modestes, mesurant $155 \times 165$ $\mathrm{mm}$ et ne comportant aucune enluminure. Le cadre de justification est de 60 x $98 \mathrm{~mm}$ et la réglure est tracée à l'encre violette. L'écriture s'étend sur une colonne de vingt lignes, avec des rubriques rouges. Les sections principales

6. Dyonisios Hatzopoulos, Dossier de recherche, Université McGill, Livres rares et collections spécialisées, s.d. ; Seymour de Ricci et Jerome Wilson, Census of Medieval and Renaissance Manuscripts in the United States and Canada (New York : The H. Wilson Company, 1937), II : 2203-2222. 
sont introduites par une initiale peinte, dorée et encadrée par une bordure végétalisée dans les quatre marges. Les sections secondaires sont introduites par une simple rubrique et signalées par cette même bordure végétalisée. Nous retrouvons aussi des initiales peintes et dorées d'une, deux et trois lignes de haut, ainsi que des initiales ornées et rehaussées d'or sur fond rouge ou bleu. Dans la litanie des saints, les bouts de lignes sont ornés.

\subsection{Considérations générales sur le décor gothique}

Le décor monastique des reliures du XIII ${ }^{e}$ aux XV ${ }^{e}$ siècles est le terme définissant l'ensemble des fers ou fleurons qui les rendent aisément reconnaissables ${ }^{7}$. Ces fleurons sont de forme géométrique, soit ronde, carrée ou en losange et appliqués dans des compartiments symétriques sur les plats. Ce qui différencie les fleurons des XII ${ }^{e}$ aux XVe siècles de ceux des XVI ${ }^{e}$, XVII ${ }^{e}$ et XVIII ${ }^{e}$ siècles est le fait que les motifs ornementaux des premiers sont gravés en creux dans le métal des fleurons, alors que les motifs des deuxièmes sont gravés en relief dans le métal. Évidés, les fers sont gravés en creux. Le motif dont le cuir épousera la forme, apparaitra alors, en relief, pour donner un gaufrage ou, ce que l'on appelle aujourd'hui, l'estampage. Cette technique, aussi appelée travail au froid par les doreurs-relieurs, implique que l'on humidifie le cuir avec de l'eau, puis que l'on pose le fleuron, chauffé à une certaine température, sur la peau. Quand il est réussi, un fleuron de style monastique est la reproduction, légèrement en creux, du motif gravé en relief sur l'outil ; cette reproduction sur le cuir sera d'un ton brun foncé, lisse et brillante (sans l'ajout de feuille d'or ou d'argent, procédé qui, au $\mathrm{XV}^{\mathrm{e}}$ siècle, reste encore à être inventé). Il existe aussi une grande analogie avec les thèmes qu'illustrent les fers de cette période. Ce sont généralement "des entrelacs ornementaux ou des motifs religieux, des animaux réels ou fantastiques, des éléments de la faune, et souvent aussi, des motifs empruntés à d'autres arts décoratifs tels sculptures de façades, de portails, de meubles, gravures des sceaux, vitraux et ouvrages de ferronnerie. Ce qu'ils représentent évoque la société médiévale, ses légendes et ses croyances ${ }^{8}$. Les filets et les fleurons sont disposés selon un schéma que l'on retrouvera sur de nombreuses reliures du Moyen Âge : ils forment plusieurs compartiments

7. Yves Devaux, Dix siècles de reliure (Paris : Pygmalion, 1981), 24.

8. Devaux, 30. 
rectangulaires, de dimensions décroissantes, enfermés les uns dans les autres, mis bout à bout, pour former de grandes bandes. Enfin, l'exécution de ces décors peut surprendre parce que presque toujours malhabile, car les fers sont poussés de travers ou disposés irrégulièrement. Le rapport aux proportions et à la perspective au Moyen Âge est bien différent du nôtre.
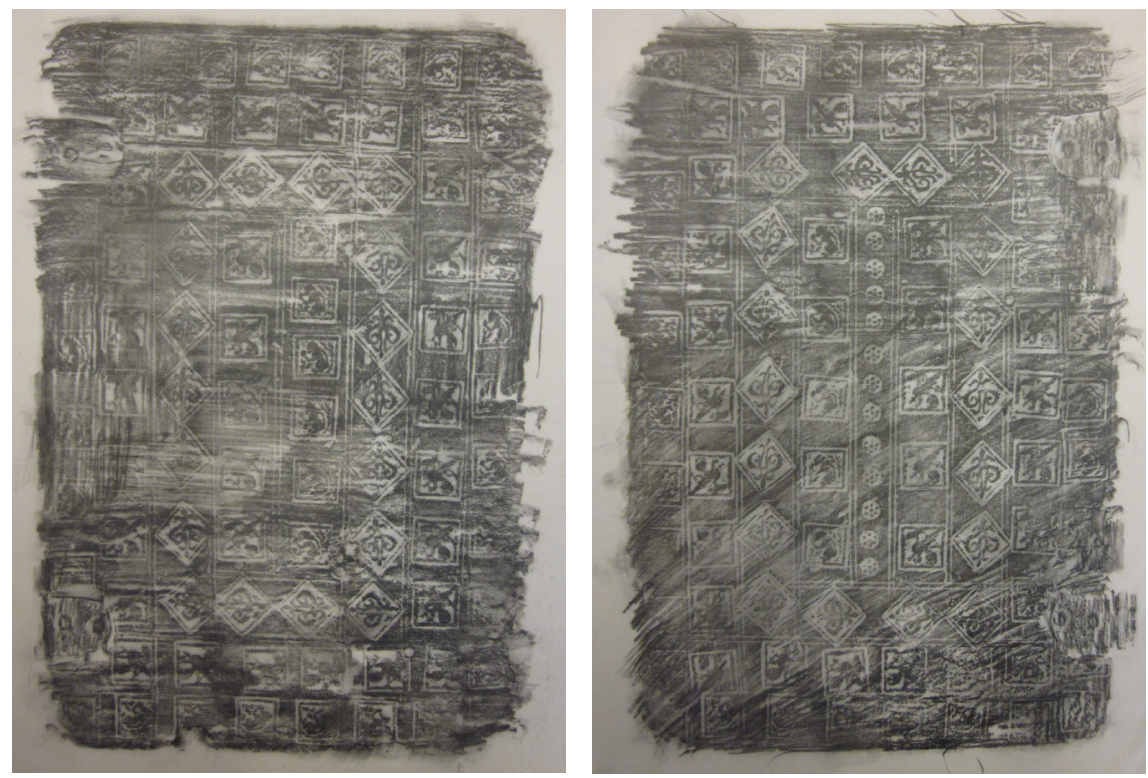

Fig. 3. Frottis des plats supérieur et inférieur du manuscrit McGill, MS 101.

\subsection{Le décor du manuscrit McGill, MS 101}

Les deux plats de la reliure du manuscrit McGill, MS 101 sont décorés de filets à l'ancienne et de quatre fers de forme géométrique : un rond, deux carrés et un losange. Le dos est, quant à lui, décoré de filets doubles disposés en losange sans aucun fer décoratif. Pour faciliter la description, les plats ont été divisés en quatre compartiments, le premier compartiment commençant par le compartiment central. Le compartiment central du plat supérieur est divisé en trois colonnes et composé de trois fers, deux carrés et un rond, posés verticalement. Au centre de ce compartiment est posé treize fois un petit fer rond de $4 \mathrm{~mm}$ représentant une fleur à six pétales. Dans la colonne de gauche, se trouve un premier fer 
carré de $12 \mathrm{~mm}$, posé cinq fois, représentant un singe de profil, accroupi, tourné vers la gauche, et on remarque le détail du feuillage autour de l'animal. Dans la colonne de droite, un autre fer carré de même dimension, représentant une vouivre, wyvern en anglais, est posé comme le singe, cinq fois. La vouivre, une créature fantastique, porte une escarboucle sur le front, un œil fait d'un charbon ardent ou d'une pierre précieuse. Notre fer la représente possédant deux pattes, deux ailes et une queue hérissée de pointes. Comme le singe, elle est de profil, assise et également tournée vers la gauche. Dans le compartiment suivant, est posé dix-huit fois, quatre fois à l'horizontal et cinq fois à la verticale, le quatrième fer, en forme de losange, de $12 \mathrm{~mm}$, représentant la fleur de lys.

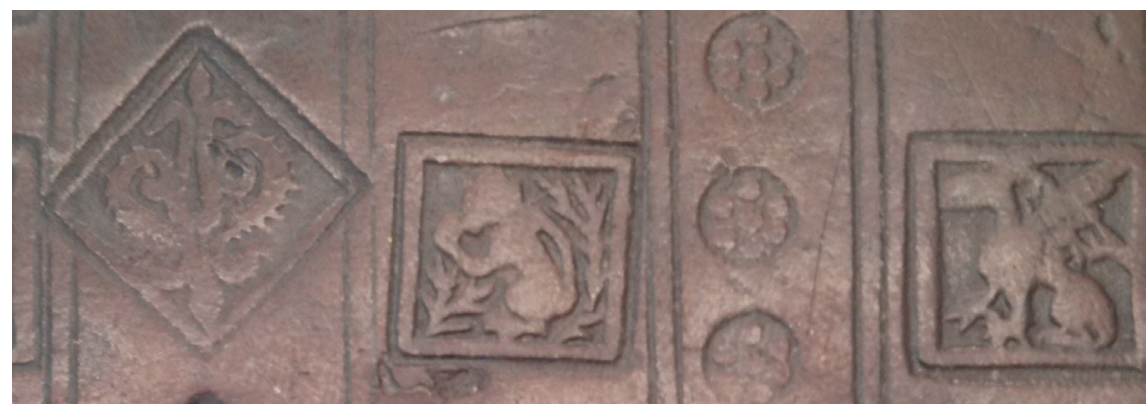

Fig. 4. Les fers monastiques du manuscrit McGill, MS 101.

Dans les deux derniers compartiments, en marge des plats, sont disposés à nouveau, vingt-six fois horizontalement et verticalement, la vouivre et enfin, trente-quatre fois, le singe. Le plat inférieur est presque à l'identique quant au nombre des compartiments si ce n'est que le compartiment central ne comprend que deux colonnes au lieu de trois, le petit fer rond floral n'étant pas utilisé. Lordre et la composition des trois autres compartiments restent les mêmes que ceux du plat supérieur. Il en découle pour le plat supérieur une harmonie plus grande que pour le plat inférieur, par son cadre à trois compartiments entourant une partie centrale de trois colonnes. On peut sans doute conclure que le plat supérieur a davantage d'importance, parce qu'il est plus visible lorsque le livre est posé, et que le relieur ménage un peu ses efforts pour le plat inférieur. Les colonnes par trois ont, sans doute, une valeur symbolique. 
2.3.1 Couvrure, fermoirs, couture et tranchefiles

\section{La couvrure}

Les reliures de cuir de cette époque, parvenues intactes jusqu'à nous, sont rares. Le manuscrit McGill, MS 101 constitue un magnifique témoignage des reliures de cuir de cette époque, dans son état d'origine. La couvrure est de veau, de couleur brun foncé. Comme les ouvrages de cette période, il ne possède pas de pièces ou d'étiquettes-titres, ni sur les plats, les tranches ou le dos. Les ais de bois sont minces, les chasses - cet espace sur le contreplat qui dépasse du volume en tête, en queue et en gouttière - sont apparentes. Les coins, ou le morceau central de cuir est replié jusqu'au bord des ais : deux coupes sont pratiquées, ne laissant subsister au centre qu'une languette de cuir qui protège l'angle des ais. La réfection des coiffes a été nécessaire pour remplacer les pièces manquantes. Elles ont été restaurées avec un cuir à l'identique, soit une peau de veau foncée. Dernièrement, le corps d'ouvrage a été repositionné dans sa reliure d'époque et repose maintenant dans une boite de protection faite sur mesure.

\section{Les fermoirs}

La préparation rudimentaire des mors rendait nécessaire les fermoirs, surtout quand il s'agissait de feuillets parchemins, car sous l'action de l'humidité, le parchemin se gondolait, et les plats avaient tendance à bailler. Les fermoirs du manuscrit McGill, MS 101 sont de laiton torsadé. Le départ de l'agrafe se fait du plat supérieur. Autre constatation : sur le plat supérieur, la présence de trois trous formant un triangle. S'il s'agit de traces de fermoirs antérieurs, nous pouvons nous interroger sur l'absence de pareilles traces des contre-agrafes au plat inférieur.

\section{La couture}

Selon le dossier de restauration, nous savons que ce livre d'Heures a été restauré en 1996 par ce même restaurateur des manuscrits McGill, MS 100, MS 109 et MS 156. Pour le McGill, MS 101, la couture d'origine n’a pas été maintenue, le volume a été décousu. La nouvelle couture, montée sur trois lanières doubles de cuir, a été refaite à l'identique et les nerfs saillants en relief sur le dos ont été fouettés au moment de la pose de la nouvelle couvrure. Les contre-gardes du manuscrit McGill, MS 101 sont toujours intactes, mais elles sont évidemment très salies ou abîmées, car ce sont des parties qui ont été très exposées aux 
manipulations au cours des quatre derniers siècles. Elles ont été soulevées en gouttière pour permettre l'insertion de nouveaux demi-feuillets en parchemin. Ces demi-feuillets ont été cousus au texte.

\section{Les tranchefiles}

Les tranchefiles de notre manuscrit étaient à l'origine tressées sur une lanière de cuir, avec un point décoratif créant une ornementation maintenant difficile à identifier. Elles sont montées sur un septain de chanvre, un simple bâti sans point décoratif, comme pour le manuscrit McGill, MS 109 présenté plus haut.

\subsection{La collation des cahiers et l'analyse codicologique du manuscrit McGill, MS 101}

La collation des cahiers consiste à déterminer les limites des différents cahiers, leur type et leur succession en confrontant les signes et les éléments mentionnés ci-haut afin de reconstituer l'état primitif d'un manuscrit. Le diagramme suivant présente la collation des cahiers du manuscrit McGill, MS 101 où l'on trouve en abscisse, les éléments suivants : le nombre et le type de cahiers, la foliotation et les feuillets. Notons que la couture se situe entre le feuillet quatre et le feuillet cinq. En ordonnée, se retrouvent les cahiers, numérotés de un à sept.

\begin{tabular}{|c|c|c|c|c|c|c|c|c|c|c|}
\hline $\begin{array}{c}\mathbf{1 .} \\
\mathbf{N}^{\circ} \mathbf{d e} \\
\text { cahier }\end{array}$ & $\begin{array}{c}\mathbf{2 .} \\
\text { Type de } \\
\text { cahier }\end{array}$ & $\begin{array}{c}\mathbf{3 .} \\
\text { Folio- } \\
\text { tation }\end{array}$ & $\mathbf{f . 1}$ & $\mathbf{f . 2}$ & $\mathbf{f . 3}$ & $\mathbf{f . 4}$ & $\mathbf{f . 5}$ & $\mathbf{f . 6}$ & $\mathbf{f . 7}$ & f.8 \\
\hline 1 & $4 / 4$ & $1-8$ & 1 & 2 & 3 & 4 & 5 & 6 & 7 & 8 \\
\hline 2 & $4 / 3^{*}$ & $9-15$ & 9 & 10 & 11 & 12 & 13 & 14 & 15 & $\mathbf{X}$ \\
\hline 3 & $4 / 4$ & $16-23$ & 16 & 17 & 18 & 19 & 20 & 21 & 22 & 23 \\
\hline 4 & $4 / 4$ & $24-31$ & 24 & 25 & 26 & 27 & 28 & 29 & 30 & 31 \\
\hline 5 & $3 / 4^{*}$ & $32-38$ & 32 & 33 & 34 & $\mathbf{X}$ & 35 & 36 & 37 & 38 \\
\hline 6 & $3 / 4^{*}$ & $39-45$ & 39 & 40 & 41 & $\mathbf{X}$ & 42 & 43 & 44 & 45 \\
\hline 7 & $4 / 4$ & $46-53$ & 46 & 47 & 48 & 49 & 50 & 51 & 52 & 53 \\
\hline
\end{tabular}

Fig. 5. Tableau de la collation des cahiers du manuscrit McGill, MS 101. 
L’analyse codicologique nous révèle que ce livre d'Heures était originalement composé de quaternions soit de vingt-huit bifolios, pour un total de cinquante-six feuillets dont cinquante-trois bifolios subsistent. Cette anomalie est confirmée par la présence d'onglets dans les cahiers deux, cinq et six. L'ensemble du codex se présente sans aucune alternance de types des cahiers. Une description rédigée se résumerait à ceci : sept cahiers, composés de quaternions, soit un quaternion, un quaternion amputé du dernier feuillet, deux quaternions, un quaternion amputé du premier feuillet médian, un quaternion amputé du premier feuillet médian et un quaternion. La description chiffrée suivrait comme suit : $1^{8}$, $2^{8}(-1), 3^{8}-4^{8}, 5^{8}(-1), 6^{8}(-1), 7^{8}$.

Il restera au cours de nos recherches à vérifier si les textes ont été copiés par un même scribe, en un même lieu et à une même époque. Alors, nous pourrons authentifier la constitution homogène ou composite de ce volume ${ }^{9}$. La critique du texte de ce manuscrit permettra d'identifier les passages manquants, sur lesquels la description codicologique attire notre attention.

\section{Conclusion}

Ce panorama descriptif des reliures des neuf livres d'Heures des collections conservées à McGill, ainsi que l'analyse codicologique détaillée du manuscrit McGill, MS 101, ont permis d'entrevoir la richesse de la dimension matérielle de ces œuvres conservées à l'Université McGill. Grâce à l'examen des reliures, nous suivons, par la même occasion, l'évolution des styles historiques, sans oublier celle des méthodes et techniques de restauration dont les goûts varient au fil des siècles, mais qui disent toujours que ces livres ont été tenus comme des objets précieux tantôt par dévotion, tantôt par bibliophilie. Somme toute, nous souscrivons à une approche globale de l'étude du livre médiéval, telle que l'a présentée Donatella Nebbiai :

Le livre médiéval est un objet archéologique, qui véhicule un ou plusieurs textes ; c'est un document chargé de renseignements historiques, puisqu'il porte les traces des hommes qui l'ont exécuté, possédé et lu. Ces marques de fabrication, d'appartenance et de lecture sont nombreuses et diverses, et leur exploitation aide à retracer les étapes de l'histoire du manuscrit et de 
l'élaboration du texte. Rapprochées les unes des autres, elles contribuent à replacer le livre dans son contexte d'origine : le fonds médiéval ${ }^{10}$.

Il nous semble que la description physique des caractéristiques de la fabrication des reliures d'un manuscrit, selon une approche codicologique, s'insère dans cette approche globale de l'étude du livre médiéval tout comme elle peut ouvrir de nouvelles pistes pour l'histoire des livres d'Heures et celle du livre médiéval. 